\title{
The Role of Extended Prostate Biopsy on Prostate Cancer Detection Rate: A Study Performed on the Bench
}

\author{
Luciano Nesrallah, Adriano Nesrallah, Alberto A. Antunes, Katia R. Leite, Miguel Srougi \\ Division of Urology, University of Sao Paulo Medical School, USP, Sao Paulo, SP, Brazil
}

\begin{abstract}
Introduction: The aim of this prospective study was to compare the advantage of performing prostate biopsy with a greater number of cores using the classic sextant procedure, with the aim of reducing false negative results.

Materials and Methods: 100 prostates were acquired from consecutive radical prostatectomies performed by the same surgeon. Fourteen cores were obtained on the bench following surgery using an automatic pistol with an 18-gauge needle. Six of these cores were obtained according to the sextant technique, as described by Hodge et al.; with the addition of a further three lateral cores from each lobe and one from the bilateral transition zone. The whole gland and the fragments were assessed by the same pathologist. An analysis of the frequency of the cancers identified in the cores of the sextant and the extended biopsies was undertaken and the results evaluated comparatively. The chi-square test was used for the comparative analysis of the cancer detection rate, according to the technique used.

Results: When 6 cores were removed, the positive cancer rate was $75 \%$, which was increased to $88 \%$ when 14 cores were $(\mathrm{p}<0.001)$. The withdrawal of 14 cores resulted in a significant $13 \%(95 \% \mathrm{CI}[5 \%-21 \%])$ increase in the positive rate of cancer detection.

Conclusion: Extended biopsy, with the removal of 14 cores, is more efficient than the sextant procedure in improving the rate of prostate cancer detection.
\end{abstract}

Key words: prostatic neoplasm; biopsy needle; pathology; diagnosis

Int Braz J Urol. 2008; 34: 563-71

\section{INTRODUCTION}

Prostate cancer is the most common non-cutaneous malignant tumor in men. In the United States, it accounts for $33 \%$ of all new cancer cases and it is estimated that 218,890 men will be diagnosed in 2006, $91 \%$ of which will be discovered at a localized or regional stage. Moreover, it is estimated that 27,050 men will die of the disease (1).

A great challenge for the early diagnosis of prostate cancer is that in its initial phase, the tumor is asymptomatic and only detected by the alterations in the digital rectal examination, abnormal increase in the plasma level of the prostate specific antigen
(PSA) or by means of transrectal ultrasound revealing hyperechoic and hypervascularized areas. When any of these alterations is found, it becomes necessary to perform a transrectal ultrasound-guided prostate biopsy.

In 1989, Hodge and colleagues proposed the undertaking of routine sextant biopsy and demonstrated the superiority of the method compared to the digitally guided biopsy directed to nodules or suspected areas. This method has become the gold standard for the diagnosis of prostate cancer (2).

A study with a mathematical laboratory model showed that sextant biopsy could detect the tumor in $36 \%, 44 \%$ and $100 \%$ of the cases in which 
the lesion occupied $2.5 \%, 5 \%$ and $20 \%$, respectively, of the gland volume (3). However, the sextant biopsy has led to false negative results in $15 \%$ to $34 \%$ of men (4-7). Furthermore, the general sensitivity of the sextant biopsy was only $60 \%$ in patients with normal prostatic DRE (8).

For these reasons, various modifications of the technique have been proposed, some of which suggested the acquisition of cores in a more lateral region (9) or an increase in the number of cores obtained (10-13). There seems to be a consensus in the literature as to the superiority of the techniques that involve the withdrawal of a larger number of cores for the diagnosis of adenocarcinoma of the prostate $(10,12,14)$. However, the number of cores to be obtained varies significantly among the various studies published, with no overall consensus. In reviewing the subject, we found only one prospective and randomized study that compared 6 and 12-core biopsies obtained in a significant number of patients (14). This study did not demonstrate any significant difference between the two procedures in the detection of prostate cancer.

Curiously, recent analyses have shown that in the United States and the United Kingdom, traditional sextant biopsy continues to be used in $20 \%$ to $70 \%$ of the diagnostic centers, demonstrating a general uncertainty as to the ideal number of cores to be obtained in prostate biopsy (15-17). In the studies that show advantages from the removal of more than 6 cores, the comparison of sextant with extended biopsy was performed in groups of patients suspected of having of prostate cancer. To examine the true incidence of false negative results, it would be necessary to obtain the biopsy on patients known to be bearers of the disease (i.e., those with a previous positive biopsy). Further, the withdrawal of a large number of cores could lead to an increase in the diagnosis of tumors of no clinical importance and requiring no treatment. This question could only be answered if the entire prostate were examined histologically and completely, thus permitting the definition of the pathological characteristics of the tumor.

This study compares the rates of prostate cancer detection by means of the withdrawal of 6 or 14 cores in order to determine the cancer detection rates of the two techniques.

\section{MATERIALS AND METHODS}

The study comprised of a prospective and controlled analysis of prostate glands obtained by radical prostatectomy of one hundred consecutive patients with clinically localized prostate cancer during the period of August 2000 to March 2001. The project was approved by the Ethics in Research Committee of the hospital.

All the operations were performed by the same surgeon (MS), in accordance with the surgical technique previously described.

The inclusion criteria were the presence of adenocarcinoma of the prostate in clinical stages T1T2 and recommendation of radical prostatectomy. Patients with a previous history of radiotherapy, hormone therapy or transurethral removal of the prostate were excluded.

All the surgical specimens were subjected to biopsy on the bench, immediately after their removal with an 18-gauge needle and an automatic pistol. Six cores were obtained from the peripheral zone, one from each sextant, according to Hodge et al. and were identified and analyzed under the label sextant biopsy (2). An additional 8 cores were then removed, three from the peripheral zone of the most lateral edge of the prostate and one core from the transitional zone of each lobe (Figures-1 and 2). These cores were analyzed together with the first 6 (a total of 14 cores) and were labeled as extended biopsy. All the cores were stored in separate flasks and sent, together with the surgical specimens, for pathological analysis. The histological reading of all the slides was carried out by the same pathologist (KRL).

The biopsy cores and the surgical specimens were fixed in $10 \%$ formalin for a period of 4 to 16 hours. All the glands were submitted to histological study in accordance with the recommendations previously described (18).

Both the biopsy cores and the surgical specimens were investigated for the presence of adenocarcinoma, as classified in accordance with Gleason's criteria (19).

The chi-square test was used for the comparison of the frequency of the positive results in both the sextant and the extended biopsies, employing a $95 \%$ confidence interval $(95 \% \mathrm{CI})$ for each value. A 



Figure 1 - Sextant biopsy.
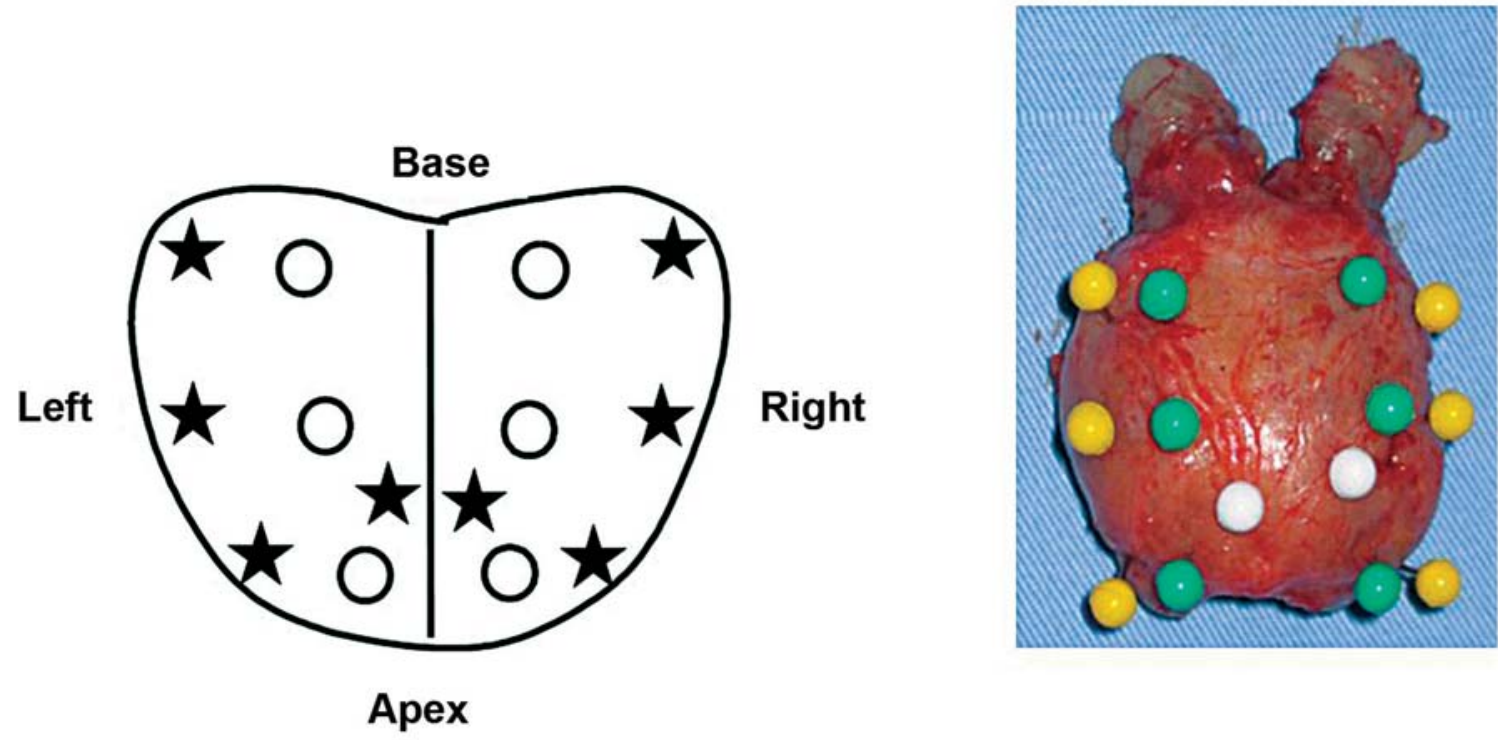

Figure 2 - Extended biopsy. 
$5 \%$ significance level $(\mathrm{p}<0.05)$ was adopted for the rejection of the null hypothesis.

\section{RESULTS}

The determined positive cancer rate was compared between the groups of the sextant and extended biopsies. Each of the specimens assessed was considered to be positive when at least one positive core was found in the sample. Table- 1 shows the joint distribution of the 100 assessed specimens when six and 14 cores were analyzed.

It may be observed from Table-1 that when 6 cores were removed the positive cancer rate was of $75 \%$, compared to a rate of $88 \%$ when 14 cores were removed. Results of McNemar's chi-squared test showed that there was a difference in the positive detection rate between these two techniques ( $p$ $<0.001$ ). Thus, when 14 cores were removed there was a significant increase in the positive cancer detection rate (Figure-3), estimated at $13 \%(95 \%$ CI [ $5 \%$ $-21 \%])$.
Cancer detection in each group increased as PSA increased but there was no significant difference between the groups (Table-2).

\section{COMMENTS}

Transrectal ultrasound-guided biopsy is the procedure of choice for the diagnosis of prostate cancer when the disease is suspected due to alterations in the PSA levels and/or alterations perceived by the DRE. This method has been modified, as there has recently been a tendency to obtain more than 8 cores, despite a lack of standardized literature on the subject. Regardless of recent studies demonstrating that extended biopsy fails less often in the diagnosis of prostate adenocarcinoma compared to the classic sextant biopsy proposed by Hodge et al., a large number of clinics still prefer the sextant technique. Although no data on this subject has been reported, we observed that the majority of clinics continue to follow the sextant method and biopsy only 6 cores. In our study, we were able to demonstrate that 6 cores

Table 1 - Distribution of the 100 surgical specimens assessed by biopsy.

\begin{tabular}{llccc}
\hline & & \multicolumn{2}{c}{ Sextant $(6$ cores $)$} & Total \\
& & Positive & Negative & \\
\hline Extended & Positive & 75 & 13 & 88 \\
$(14$ cores $)$ & Negative & 0 & 12 & 12 \\
& Total & 75 & 25 & 100 \\
\hline
\end{tabular}

Table 2 - Cancer detection rates stratified by total PSA.

\begin{tabular}{|c|c|c|c|c|}
\hline & \multicolumn{4}{|c|}{ PSA $(n g / m L)$} \\
\hline & $\leq 4$ & 4.1 a 10 & $>10$ & Total \\
\hline 6 cores & $6(54.5 \%)$ & $52(83.9 \%)$ & $16(66.7 \%)$ & $74(76.3 \%)$ \\
\hline \multirow[t]{2}{*}{14 cores } & $9(81.8 \%)$ & $58(93.5 \%)$ & $19(79.2 \%)$ & $86(88.7 \%)$ \\
\hline & $\mathrm{p}=0.250$ & $\mathrm{p}=0.061$ & $\mathrm{p}=0.250$ & \\
\hline
\end{tabular}

PSA = prostate specific antigen 


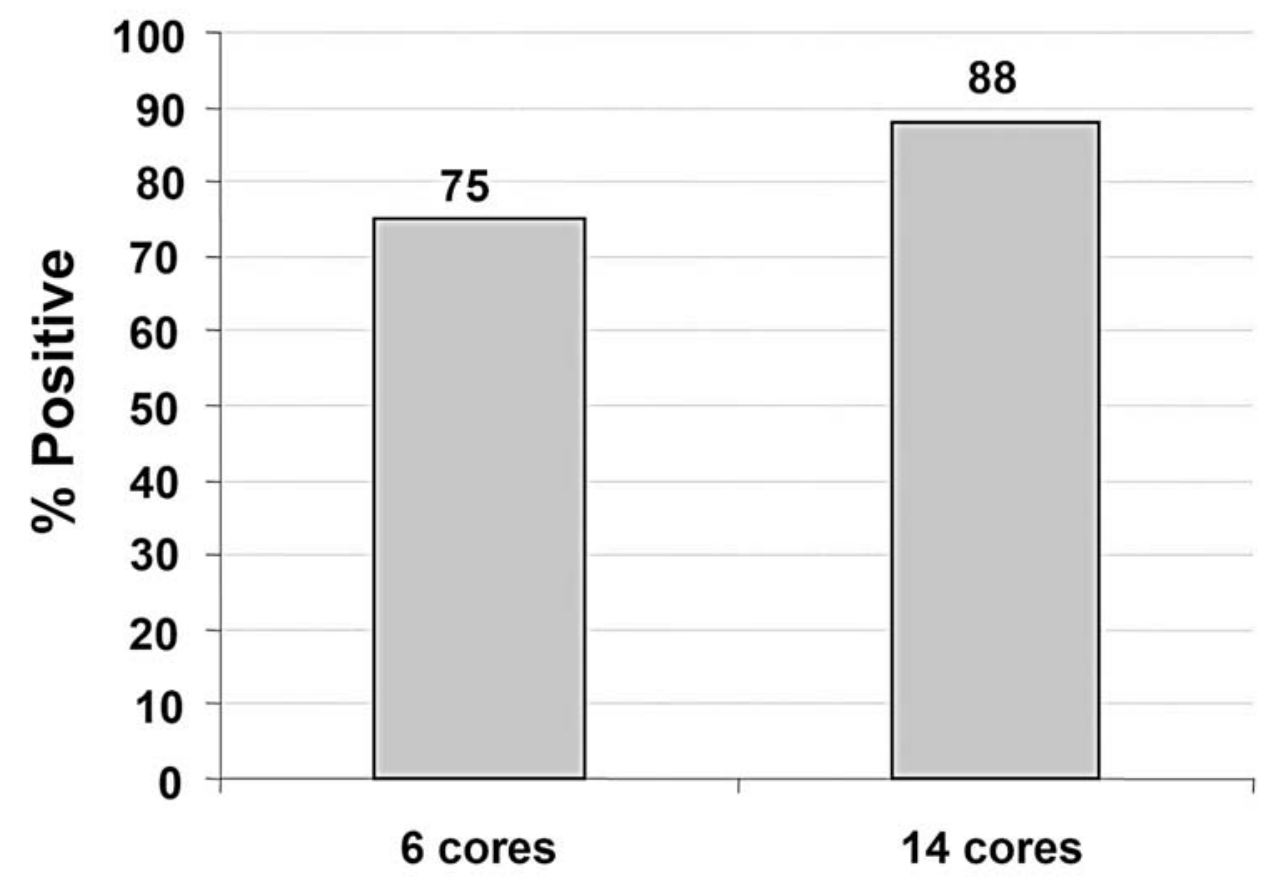

Figure 3 - Percentage of positive detection following assessment of either 6 or 14 cores isolated from 100 prostate specimens.

biopsy fails in $25 \%$ of the cases, while taking 14 cores decreases the failure rate to $12 \%$, i.e. it detects $50 \%$ more.

When the 25 prostates, where the sextant biopsy was negative, were further analyzed, it was observed that the tumor was confined in $72 \%$ of them. Although no tumors were found in any of the cores, the disease was already extraprostatic in $28 \%$ of the cases. Conversely, in those cases where no tumor was found in the 14-core biopsy, only $8.3 \%$ of the specimens showed non-localized disease.

The fact that the present study was undertaken on 100 prostates acquired from consecutive radical prostatectomies not only eliminates any possible bias in the selection of cases, but also allowed us to compare the results of the two different methods applied to the same specimens. This differentiates this study from previous reports, all of which compared the findings of sextant biopsy with those of extended biopsy, undertaken "in vivo", and therefore, on different patients. Another advantage in using surgical specimens on the bench is that the procedure permits the acquisition of cores of better quality from more precise locations, as well as being undertaken by the same investigator, which is in contrast to the published studies in which the biopsy may be taken by different doctors, introducing a possible bias. Another strength of this study is that all the operations were carried out by the same surgeon, as well as all the pathological evaluation was performed by the same uropathologist, thus avoiding the inter-observer differences that are well-known in the literature.

Because it is intuitive, it is easy to accept that an increase in the number of cores obtained in a prostate biopsy will result in an improvement in the detection rate for prostate cancer. However, there is only indirect evidence and that expectation has not yet been completely proven by scientific studies. For example, the same author suggests opposite results. Naughton and colleagues first showed that $44 \%$ of cancer diagnoses require a biopsy from 7 to 13 cores, in a retrospective analysis (20). Later in a prospective study these authors showed that there was no statistically significant difference in the diagnosis of prostate 
adenocarcinoma when 6 and 12-core biopsies were compared (14). Studies undertaken on Japanese men showed a much smaller improvement of $7.7 \%$ to $13.8 \%$ when 12 cores were obtained, as compared to those demonstrated by western studies $(21,22)$. In a prospective study, in which 6 and 12-core biopsies by transperineal approach were compared, no significant difference was found when a palpable nodule or lesion suspected by transrectal ultrasound existed, except in situations in which there was an increase in the level of PSA with no alteration in the DRE or ultrasound, when the 12-core biopsy demonstrated an advantage (23). One variable that may affect the results of the biopsy is the volume of the prostate. Cancer detection in prostates less than $50 \mathrm{cc}$ was $38 \%$, whereas prostates more than $50 \mathrm{cc}$ have a lower detection rate (23\%) (24). One randomized prospective study investigated the influence of the increase in the number of cores as a function of prostate volume. In patients with no palpable nodule but increased PSA levels, the cancer detection rate doubled when using biopsies of 10 to 14 cores compared to the sextant technique (25). This correlation involving the need to increase the number of biopsy cores in accordance with the volume of the prostate has also been found in a Turkish study, in which the authors suggest that the sextant biopsy was not reliable even in small prostates. The study further recommended that prostates of more than 35 cc should have 10 cores removed, and that in smaller glands only 8 cores need to be obtained (26). The same author had previously demonstrated, though without relating the number of cores to prostate volume, that when 10 cores were obtained the cancer detection rate increased by $25.5 \%$ as compared with the classic 6-core biopsy (27). In the present study the average weight of the prostates was of $42.4 \mathrm{cc}$, the standard deviation was $23.9 \mathrm{cc}$, and the increase in tumor detection was of $13 \%$ when 14 cores were used compared with a 6-core biopsy.

In another study of 179 consecutive patients who underwent radical prostatectomy, it was observed that a 12 -core biopsy detected $31.3 \%$ more cancer than the sextant technique (28). A group from the University of Vienna working with Athenian urologists in the attempt to validate a nomogram to define the ideal number of biopsy cores based on the age and prostate volume of the patient, submitted 502 men to biopsy on the basis of the criteria of the nomogram. The results were then compared with those of a control group of 1,051 patients who had previously had octant biopsy and re-biopsy when the former was negative. The disease was found in $36.7 \%$ of the patients when the Vienna nomogram was used, compared with $22 \%$ at the first octant biopsy and $10 \%$ on re-biopsy (29). This group confirmed that the sextant biopsy should only be used on patients of more than 70 years of age, who have a prostate volume of less than $40 \mathrm{~mL}$. After excluding that group of patients, the techniques that used at least 8 core samples was preferred, with the number of samples increasing in accordance with the increased weight of the gland and as the age of the patient diminished.

More recently, the possibility of using the so-called saturation biopsies, which involve the withdrawal of more than 18 cores, has been analyzed. In one study, 24 cores were each acquired from 139 selected men and the procedure was performed by two surgeons. The results were compared with a group of 87 patients who had previously undergone the 10-core technique. No significant difference was found in the detection of the disease between these two groups. The authors concluded that a 10or 12-core biopsy should be the method of choice for the first investigation in the early detection of prostate cancer (30). This type of strategy was also confirmed more recently in a systematic review of the literature, by which the present authors arrived at the conclusion that an investigation into prostate cancer using the 12-core biopsy should be considered (31).

On the basis of our findings and also on those reported in the literature, the authors suggest that there is no longer any justification, in practice, for the continued use of the sextant biopsy. Even with the extended technique, we still find an unacceptable number of biopsies with false negative results $(12 \%$, according to our data). In a future study we intend in to compare the extended with the saturation biopsy on the bench, which involves the acquisition of more than 18 cores, in an attempt to discover whether there is any advantage in this latter method in the detection of adenocarcinoma of the prostate. 


\section{CONCLUSION}

Extended biopsy, with the removal of 14 cores, could be considered more effective than the sextant procedure in improving the prostate cancer detection rate.

\section{CONFLICT OF INTEREST}

None declared.

\section{REFERENCES}

1. Jemal A, Siegel R, Ward E, Murray T, Xu J, Thun MJ: Cancer statistics, 2007. CA Cancer J Clin. 2007; 57: 43-66.

2. Hodge KK, McNeal JE, Terris MK, Stamey TA: Random systematic versus directed ultrasound guided transrectal core biopsies of the prostate. J Urol. 1989; 142: 71-4; discussion 74-5.

3. Feneley MR, Parkinson MC: Biopsy diagnosis of prostatic cancer--current areas of concern. J Clin Pathol. 1997; 50: 265-6.

4. Chen ME, Troncoso P, Johnston DA, Tang K, Babaian RJ: Optimization of prostate biopsy strategy using computer based analysis. J Urol. 1997; 158: 216875.

5. Ellis WJ, Brawer MK: Repeat prostate needle biopsy: who needs it? J Urol. 1995; 153: 1496-8.

6. Keetch DW, Catalona WJ, Smith DS: Serial prostatic biopsies in men with persistently elevated serum prostate specific antigen values. J Urol. 1994; 151: 1571-4.

7. Norberg M, Egevad L, Holmberg L, Sparén P, Norlén BJ, Busch C: The sextant protocol for ultrasoundguided core biopsies of the prostate underestimates the presence of cancer. Urology. 1997; 50: 562-6.

8. Terris MK: Sensitivity and specificity of sextant biopsies in the detection of prostate cancer: preliminary report. Urology. 1999; 54: 486-9.

9. Stamey TA: Making the most out of six systematic sextant biopsies. Urology. 1995; 45: 2-12.

10. Eskew LA, Bare RL, McCullough DL: Systematic 5 region prostate biopsy is superior to sextant method for diagnosing carcinoma of the prostate. J Urol. 1997; 157: 199-202; discussion 202-3.

11. Levine MA, Ittman M, Melamed J, Lepor H: Two consecutive sets of transrectal ultrasound guided sextant biopsies of the prostate for the detection of prostate cancer. J Urol. 1998; 159: 471-5; discussion 475-6.

12. Presti JC Jr, Chang JJ, Bhargava V, Shinohara K: The optimal systematic prostate biopsy scheme should include 8 rather than 6 biopsies: results of a prospective clinical trial. J Urol. 2000; 163: 163-6; discussion 166-7.

13. Babaian RJ, Toi A, Kamoi K, Troncoso P, Sweet J, Evans R, et al.: A comparative analysis of sextant and an extended 11-core multisite directed biopsy strategy. J Urol. 2000; 163: 152-7.

14. Naughton CK, Miller DC, Mager DE, Ornstein DK, Catalona WJ: A prospective randomized trial comparing 6 versus 12 prostate biopsy cores: impact on cancer detection. J Urol. 2000; 164: 388-92.

15. Davis M, Sofer M, Kim SS, Soloway MS: The procedure of transrectal ultrasound guided biopsy of the prostate: a survey of patient preparation and biopsy technique. J Urol. 2002; 167: 566-70.

16. Patel HR, Lee F, Arya M, Masood S, Palmer JH, Sheriff MK: A national survey of transrectal ultrasound-guided prostatic biopsies: time for a national guideline. Int J Clin Pract. 2003; 57: 773-4.

17. Eichler K, Hempel S, Wilby J, Myers L, Bachmann LM, Kleijnen J: Diagnostic value of systematic biopsy methods in the investigation of prostate cancer: a systematic review. J Urol. 2006; 175: 1605-12.

18. Bostwick DG, Foster CS: Examination of Radical Prostatectomy Specimens: Therapeutic and Prognostic Significance. In: Foster CS, Bostwick DG (eds.) Pathology of Prostate. Philadelphia, WB Saunders. 1998; pp. 172.

19. Gleason DF: Histologic grading of prostate cancer: a perspective. Hum Pathol. 1992; 23: 273-9.

20. Naughton CK, Smith DS, Humphrey PA, Catalona WJ, Keetch DW: Clinical and pathologic tumor characteristics of prostate cancer as a function of the number of biopsy cores: a retrospective study. Urology. 1998; 52: 808-13.

21. Matsumoto K, Satoh T, Egawa S, Shimura S, Kuwao S, Baba S: Efficacy and morbidity of transrectal ultrasound-guided 12-core biopsy for detection of prostate cancer in Japanese men. Int J Urol. 2005; 12: 353-60.

22. Kojima M, Hayakawa T, Saito T, Mitsuya H, Hayase Y: Transperineal 12-core systematic biopsy in the detection of prostate cancer. Int J Urol. 2001; 8: 301-7.

23. Emiliozzi P, Scarpone P, DePaula F, Pizzo M, Federico G, Pansadoro A, et al.: The incidence of prostate cancer in men with prostate specific antigen greater than 4.0 $\mathrm{ng} / \mathrm{ml}$ : a randomized study of 6 versus 12 core transperineal prostate biopsy. J Urol. 2004; 171: 197-9. 
24. Uzzo RG, Wei JT, Waldbaum RS, Perlmutter AP, Byrne JC, Vaughan ED Jr: The influence of prostate size on cancer detection. Urology. 1995; 46: 831-6.

25. Mariappan P, Chong WL, Sundram M, Mohamed SR: Increasing prostate biopsy cores based on volume vs the sextant biopsy: a prospective randomized controlled clinical study on cancer detection rates and morbidity. BJU Int. 2004; 94: 307-10.

26. Eskicorapci SY, Guliyev F, Akdogan B, Dogan HS, Ergen A, Ozen H: Individualization of the biopsy protocol according to the prostate gland volume for prostate cancer detection. J Urol. 2005; 173: 153640.

27. Eskicorapci SY, Baydar DE, Akbal C, Sofikerim M, Günay M, Ekici S, et al.: An extended 10-core transrectal ultrasonography guided prostate biopsy protocol improves the detection of prostate cancer. Eur Urol. 2004; 45: 444-8; discussion 448-9.

\section{Correspondence address:}

Dr. Miguel Srougi

Rua Peixoto Gomide, 2055/81

São Paulo, SP, 01409-003, Brazil

Fax: + $55113257-9006$

E-mail: srougi@terra.com.br

\section{EDITORIAL COMMENT}

It is commonly accepted that traditional sextant transrectal biopsy may underestimate the true tumor burden within the prostate after a whole mount specimen is available. Today as technology continues to evolve, it is imperative that clinicians have the most accurate and comprehensive information available upon which to base their recommendations. We know from large retrospective series of radical prostatectomies that traditional sextant biopsy may underestimate the true tumor extent by as much as $46.6 \%$ when the whole mount specimen is examined (1). The authors are to be commended on this prospective analysis of
28. Singh H, Canto EI, Shariat SF, Kadmon D, Miles BJ, Wheeler TM, et al.: Improved detection of clinically significant, curable prostate cancer with systematic 12-core biopsy. J Urol. 2004; 171: 1089-92.

29. Remzi M, Fong YK, Dobrovits M, Anagnostou T, Seitz C, Waldert M, et al.: The Vienna nomogram: validation of a novel biopsy strategy defining the optimal number of cores based on patient age and total prostate volume. J Urol. 2005; 174: 1256-60; discussion 1260-1; author reply 1261 .

30. Jones JS, Patel A, Schoenfield L, Rabets JC, Zippe $\mathrm{CD}$, Magi-Galluzzi C: Saturation technique does not improve cancer detection as an initial prostate biopsy strategy. J Urol. 2006; 175: 485-8.

31. Eichler K, Hempel S, Wilby J, Myers L, Bachmann LM, Kleijnen J: Diagnostic value of systematic biopsy methods in the investigation of prostate cancer: a systematic review. J Urol. 2006; 175: 1605-12.

Accepted after revision:

March 3, 2008

100 consecutive radical prostatectomy specimens performed by the same surgeon and pathology reviewed by the same pathologist. Previous investigators have demonstrated an increased yield of malignant diagnoses in vivo using extended systematic sextant biopsy of twelve cores versus traditional sextant biopsy (2). To my knowledge this study is unique, based on the fact that the needle core biopsies were obtained from the RP specimen on the bench.

Clearly, the author's data demonstrate a statistically significant increased yield of malignant diagnoses of $13 \%$ when additional lateral cores were 
obtained from each lobe and bilateral transition zone. These results further support the current trend of extended systematic sextant biopsy of 12-14 cores.

There is a subset of patients that will have negative biopsies even with the additional lateral and transitional cores, yet the PSA level may continue to rise. This particular scenario presents a diagnostic dilemma to the clinician and anxiety for the patient. In this setting, our approach is to perform stereotactic transperineal prostate biopsy (STPB). Similar to a prostate brachytherapy procedure, the prostate is positioned on the implant grid. Specimens are obtained according to $\mathrm{x}, \mathrm{y}$, and $\mathrm{z}$ coordinates from eight equal octants with pathology reported accordingly. Using this technique, we have consistently achieved a 39\% positive biopsy rate. There was a significant difference in detection rates with the apex having a higher incidence of malignancy than the base of the prostate gland $(p=0.000)$. Furthermore, the anterior apex harbored significantly more adenocarcinoma when compared to the posterior apex $(\mathrm{p}=0.026)(3)$.
As our knowledge of biopsy techniques become more sophisticated, it will be possible to map malignant versus benign regions of the prostate; whether or not targeted focal therapy results from this remains to be determined.

\section{REFERENCES}

1. Fukagai T, Namiki T, Namiki H, Carlile RG, Shimada M, Yoshida H: Discrepancies between Gleason scores of needle biopsy and radical prostatectomy specimens. Pathol Int. 2001; 51: 364-70.

2. Presti JC Jr, O’Dowd GJ, Miller MC, Mattu R, Veltri RW: Extended peripheral zone biopsy schemes increase cancer detection rates and minimize variance in prostate specific antigen and age related cancer rates: results of a community multi-practice study. J Urol. 2003; 169: 125-9.

3. Moran BJ, Braccioforte MH, Conterato DJ: Re-biopsy of the prostate using a stereotactic transperineal technique. J Urol. 2006; 176: 1376-81; discussion 1381 .

Dr. Brian J. Moran Medical Director, Radiation Oncology Chicago Prostate Center Westmont, Illinois, USA E-mail:seeds@prostateimplant.com 